\title{
Anti-Compton gamma spectrometry for environmental samples
}

\author{
A. de Vismes, R. Gurriaran and X. Cagnat \\ IRSN, LMRE, Bâtiment 501, Bois des Rames, 91400 Orsay, France
}

\begin{abstract}
The results of the low-level performance of an anti-Compton device are presented. The anti-coincidence action of the NaI detectors lowers the total background of the HPGe detector integrated on the energy range $30 \mathrm{keV}$ to $2730 \mathrm{keV}$ by a factor 8.1 and suppresses the continuum above $300 \mathrm{keV}$ by a factor larger than 10 . Qualitative and quantitative results on environmental samples are described to illustrate the improvement of uncertainties determination and the significant reduction of detection limits. This provides ever more accurate and precise data on natural and anthropogenic radionuclides often used as tracers for environmental studies.
\end{abstract}

\section{INTRODUCTION}

The decreasing radioactivity levels found in environmental samples require an improvement of the detection limits of gamma spectrometry. The radioactive background of a gamma spectrometry system can be reduced by reducing the amount of radionuclides present in the close surrounding of the germanium crystal (lead shielding, choice of non-radioactive materials for the cryostat, ventilation against radon...) and limiting the interactions of the cosmic radiations (cosmic veto, underground laboratory ...).

However these measures are not effective in the case of biological and mineral matrices where the natural radioactivity of these samples $\left({ }^{40} \mathrm{~K}\right.$, uranium and thorium series) generates a Compton continuum in the gamma spectra. In the same way, for a contaminated sample, the presence of an artificial radionuclide in strong concentration might mask the presence of other radionuclides in smaller quantities. For these samples a solution consists in using an anti-Compton device.

\section{MATERIALS AND METHODS}

\subsection{Configuration of the anti-Compton device}

The principle of an anti-Compton device is well known and has been described extensively [1,2]. Our device consists of a low background N-type HPGe detector surrounded by a veto detector made of a $\mathrm{NaI}$ annulus detector (outside diameter $250 \mathrm{~mm}, 400 \mathrm{~mm}$ long) and a NaI detector on the top in order to provide a quasi- $4 \pi$ geometry around the sample (see Fig. 1). The resolution of the $280 \mathrm{~cm}^{3} \mathrm{HPGe}$ detector is $1.50 \mathrm{keV}$ FWHM at $662 \mathrm{keV}$ and $1.78 \mathrm{keV}$ FWHM at $1333 \mathrm{keV}$. The whole assembly is located inside a low-background cylindrical lead chamber of $100 \mathrm{~mm}$ thickness $(80 \mathrm{~mm}$ low activity lead and $20 \mathrm{~mm}$ very low activity lead), $310 \mathrm{~mm}$ internal diameter, $700 \mathrm{~mm}$ height. The device is installed in the second basement of the laboratory under $3 \mathrm{~m}$ of borated concrete in order to reduce the contribution of cosmic rays and particularly of neutrons. It is interesting to note that by construction the anti-Compton device also works as an anti-cosmic system.

The electronics configuration is described in the Figure 1(b). The HPGe detector signal is sent to two parallel lines, the normal one and the anti-Compton one: each one is composed of a spectroscopy amplifier (Spec. Ampl.), an Analog to Digital Converter (ADC) and an Acquisition Interface Module 


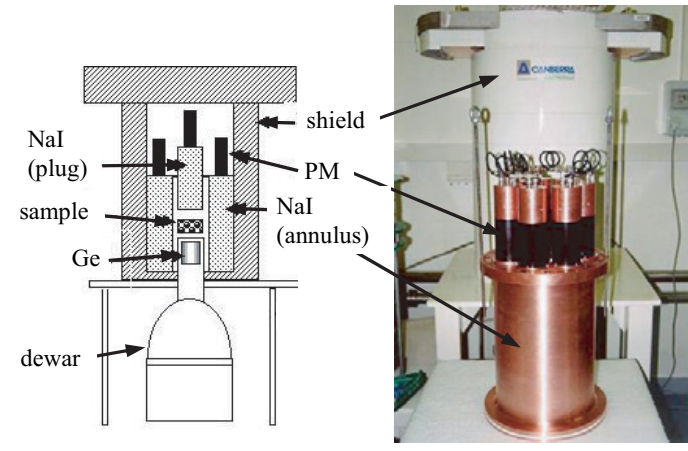

(a)

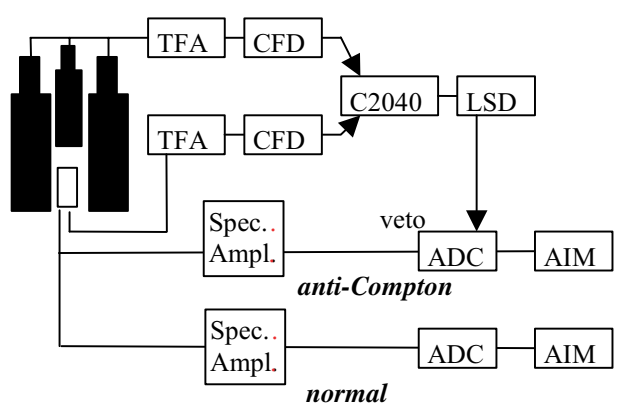

(b)

Figure 1. Anti-Compton device (a) and its electronics configuration (b).

(AIM Canberra). The veto signal sent as a gate to the ADC of the anti-Compton line is produced from the HPGe detector signal together with those from $\mathrm{NaI}$ by the anti-coincidence electronics composed of 2 Timing Filtering Amplifiers (TFA), two Constant Fraction Discriminators (CFD), a coincidence logic unit (Canberra 2040) and a Logid Shape and Delay (LSD).

Thanks to these 2 lines, two spectra are simultaneously recorded for each measurement:

- the normal spectrum that does not take into account any coincidence information,

- the Compton-suppressed spectrum where events counted in coincidence in GeHP and NaI detectors are used to reject simultaneous events in the Ge detector.

\subsection{Radionucleides of interest}

Anti-Compton device is perfectly suited for radionuclides emitting single $\gamma$-rays, that is $\beta^{-}$emitters with a simple daughter level scheme. Unfortunately decay rises very often to emission of several simultaneous photons: $\gamma$-rays emitted by cascade decaying radionuclides, $511 \mathrm{keV}$ photons emitted by $\beta^{+}$emitters and $\mathrm{X}$-rays emitted when electron capture decay; in the last case, the anti-Compton device can be used only if X-rays energy is low enough.

Study of nuclear data and decay schemes allows to classify the main radionuclides of interest in two categories:

- Radionuclides to be analysed on the anti-Compton spectrum: ${ }^{7} \mathrm{Be},{ }^{40} \mathrm{~K},{ }^{54} \mathrm{Mn},{ }^{57} \mathrm{Co},{ }^{58} \mathrm{Co},{ }^{65} \mathrm{Zn},{ }^{137} \mathrm{Cs}$, ${ }^{210} \mathrm{~Pb},{ }^{234} \mathrm{Th}$ and ${ }^{241} \mathrm{Am}$.

- Radionuclides to be analysed on the normal spectrum: ${ }^{22} \mathrm{Na},{ }^{60} \mathrm{Co},{ }^{106} \mathrm{Rh},{ }^{110 \mathrm{~m}} \mathrm{Ag},{ }^{124} \mathrm{Sb},{ }^{134} \mathrm{Cs},{ }^{154} \mathrm{Eu}$ and ${ }^{228}$ Ac.

\section{RESULTS}

The anti-Compton system has been used to measure the different families of samples found in radiological studies: biological matrices with ${ }^{40} \mathrm{~K}$ continuum (vegetables, grass, mollusks . . .), biological matrices with ${ }^{137} \mathrm{Cs}$ contamination (mushrooms...), environmental samples with $\mathrm{U}$ and Th natural radioactivity (sediments ... ) and aerosol filters with ${ }^{7} \mathrm{Be}$ Compton continuum or ${ }^{137} \mathrm{Cs}$ contamination.

In presented results all activities are given with only statistical uncertainties at $\pm 2 \sigma$ confidence level and detection limits are given by Genie2000 software with the GTN5 formulae [3]. 


\subsection{Background spectra}

The normal background spectrum (black line on Figure 2, total counting time of $2 \times 10^{6}$ seconds) shows the significance of the cosmic continuum in the spectrum which is produced by the penetrating cosmic ray muons and their secondary particles [4]. The most important line is the $511 \mathrm{keV}$ line due to the positron annihilation. Other peaks are the sign of the contamination of the detector and its sourrounding by natural radionucleides such as ${ }^{40} \mathrm{~K}(1460 \mathrm{keV}),{ }^{238} \mathrm{U}$ and ${ }^{232} \mathrm{Th}$ decay products (e.g. ${ }^{214} \mathrm{Bi}$ at $1764 \mathrm{keV}$ and ${ }^{208} \mathrm{Tl}$ at $\left.2614 \mathrm{keV}\right)$.

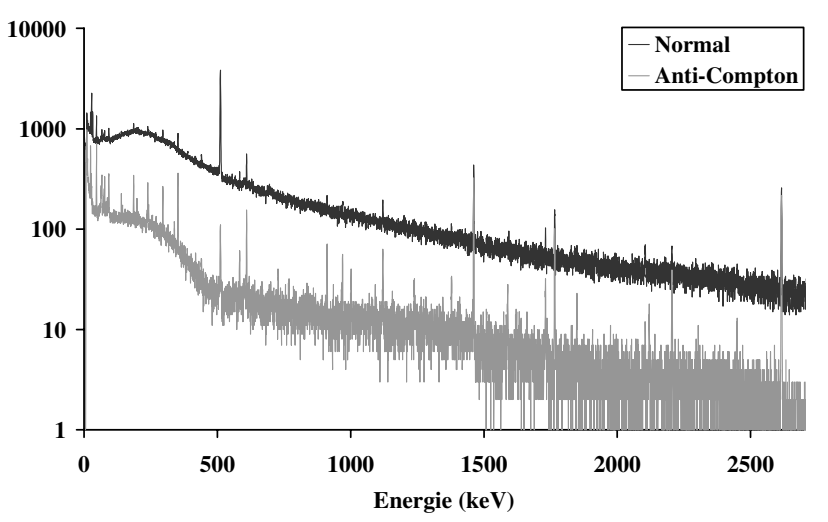

Figure 2. Normal (black line) and Compton-suppressed (gray line) background spectra.

The reduction factor RF is defined as the ratio:

$$
\mathrm{RF}=\frac{\mathrm{n}_{\mathrm{N}}}{\mathrm{n}_{\mathrm{AC}}}
$$

with:

$\mathrm{n}_{\mathrm{N}}=$ count rate without the anti-Compton system, i.e. on the normal spectrum.

$\mathrm{n}_{\mathrm{AC}}=$ count rate wit the anti-Compton system, i.e. on the Compton suppressed spectrum.

The annihilation line is suppressed the most in the anti-Compton mode: it reduces its intensity by a factor of around 42 showing the interest of this "anti-cosmic" device.

$\mathrm{RF}$ is equal to 1 for ${ }^{210} \mathrm{~Pb}$ at $46.5 \mathrm{keV}$ and for ${ }^{40} \mathrm{~K}$ at $1461 \mathrm{keV}$ : it means that there is no count losses when using the anti-Compton system and confirm that the analysis of these two radionuclides can be performed with the Compton-suppressed spectrum. On the contrary RF values of 2.2 for the ${ }^{214} \mathrm{Bi}$ line at $609 \mathrm{keV}$ and 1.8 for ${ }^{228} \mathrm{Ac}$ at $969 \mathrm{keV}$ confirm that the activities of these radionuclides have to be calculated from the normal spectrum.

Moreover the reduction factors calculated on the continuum by energy range vary from 5 at low energy $(<100 \mathrm{keV})$ to more than 20 around $600 \mathrm{keV}$. This continuum reduction suggest that an improvement of the detection limits of a factor 4 can be obtained. This would be achieved with 4 times longer counting times, incompatible with routine measurements.

Finally the total count rate integrated on the energy interval from $30 \mathrm{keV}$ to $2730 \mathrm{keV}$ is reduced 8.1 times reaching $0.10 \mathrm{c} / \mathrm{s}$, i.e. 6 events per minute.

\subsection{Biological matrices with ${ }^{40} \mathrm{~K}$}

The anti-Compton spectrometer is especially effective for the measurement of vegetal or marine samples where ${ }^{40} \mathrm{~K}$ usually dominates the spectrum. Indeed its wide Compton continuum complicates the analysis of radionuclides with lower energies. This is illustrated by the spectra (Figure 3a) (normal 


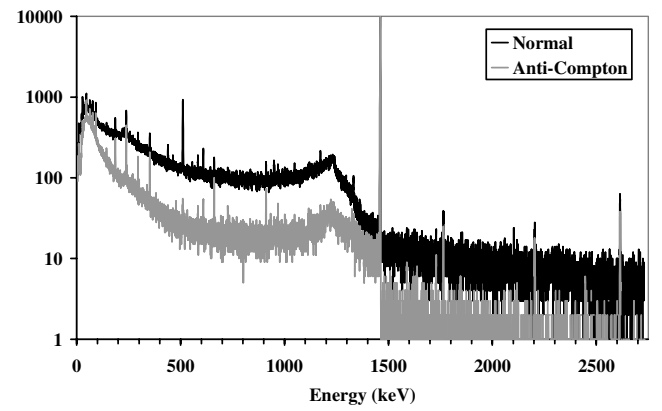

(a)

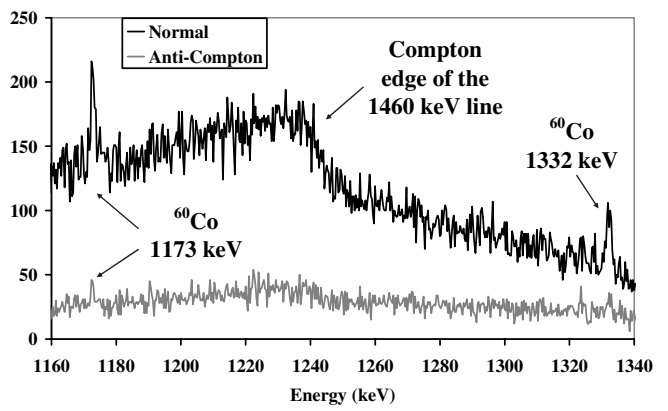

(b)

Figure 3. Spectra obtained for the measurement of mussel sample.

spectrum in black line and Compton-suppressed spectrum in gray line) obtained for a $400000 \mathrm{~s}$ measurement of a mussel sample (ashes) with an ${ }^{40} \mathrm{~K}$ activity of $1600 \mathrm{~Bq} / \mathrm{kg}$.

The effectiveness of an anti-coincidence shielding system for Compton suppression can be evaluated by measuring the suppression factor SF, with:

$$
\mathrm{SF}=\frac{(\mathrm{P} / \mathrm{C})_{\mathrm{AC}}}{(\mathrm{P} / \mathrm{C})_{\mathrm{N}}}
$$

where $(\mathrm{P} / \mathrm{C})_{\mathrm{N}}$ and $(\mathrm{P} / \mathrm{C})_{\mathrm{AC}}$ are respectively peak-to-Compton ratios in the normal and in the antiCompton spectrum. Compton plateaus heights are calculated on the edge of the Compton plateau in order not to be disturbed by other peaks.

Peak-to-Compton ratio increases from 65 to 278 with anti-Compton mode and thus the suppression factor is $\mathrm{SF}=4.3$ : that is particularly visible on the Fig. $3 \mathrm{~b}$ centered on the energy of the ${ }^{40} \mathrm{~K}$ Compton edge. This figure also shows clearly the count losses in the case of cascade decaying radionuclide: both peaks of ${ }^{60} \mathrm{Co}(1173$ and $1332 \mathrm{keV}$ ) almost disappear in the anti-Compton spectrum. Analysis of the anti-Compton spectrum gives an activity 5.3 lower than the real one: $0.15 \pm 0.08 \mathrm{~Bq} / \mathrm{kg}$ instead of $0.80 \pm 0.15 \mathrm{~Bq} / \mathrm{kg}$. This is the reason why ${ }^{60} \mathrm{Co}$ activity is always given by the analysis on the normal spectrum.

For this sample the removal of scattered photons allows to show some spectral information in the lower energy range:

- more precise determination of activities of ${ }^{210} \mathrm{~Pb},{ }^{234} \mathrm{Th}$ and especially ${ }^{137} \mathrm{Cs}$ (from $\pm 20 \%$ to $\pm 12 \%$ )

- improvement of detection limits for ${ }^{241} \mathrm{Am}$ (divided by 1.2 ), ${ }^{57} \mathrm{Co}$ (by 1.4 ) and ${ }^{58} \mathrm{Co}$ (by 2.3 )

- determination of significant values instead of detection limits for ${ }^{7} \mathrm{Be}(\leq 54 \mathrm{~Bq} / \mathrm{kg} \rightarrow 25 \pm 9 \mathrm{~Bq} / \mathrm{kg}$ ) and ${ }^{65} \mathrm{Zn}(\leq 2.0 \mathrm{~Bq} / \mathrm{kg} \rightarrow 0.83 \pm 0.29 \mathrm{~Bq} / \mathrm{kg})$.

\subsection{Samples with natural radioactivity $U$, Th}

Fig. 4a shows the spectrum obtained by measuring a sample of particles from fluvial water. The majority of the peaks is due to natural radionucleide such as ${ }^{40} \mathrm{~K}$, as well as ${ }^{238} \mathrm{U}$ and ${ }^{232} \mathrm{Th}$ decay products (e.g. ${ }^{208} \mathrm{Tl}$ at $2614 \mathrm{keV}$ ). Indeed natural radionucleides and their progenies are present with an activity concentration around $30-40 \mathrm{~Bq} / \mathrm{kg}$. Two Compton continua are present: below $1250 \mathrm{keV}$ due to ${ }^{40} \mathrm{~K}(500 \mathrm{~Bq} / \mathrm{kg})$ and due to the $2614 \mathrm{keV}$ line of ${ }^{208} \mathrm{Tl}(13 \mathrm{~Bq} / \mathrm{kg})$ with the Compton edge around $2400 \mathrm{keV}$.

In the Compton-suppressed spectrum (Fig. 4b), this Compton continuum has almost completely disappeared and the ${ }^{40} \mathrm{~K}$ Compton continuum has decreased. The suppression factors calculated for both redionucleides are respectively 5 for ${ }^{40} \mathrm{~K}$ and 2.5 for ${ }^{208} \mathrm{Tl}$. 


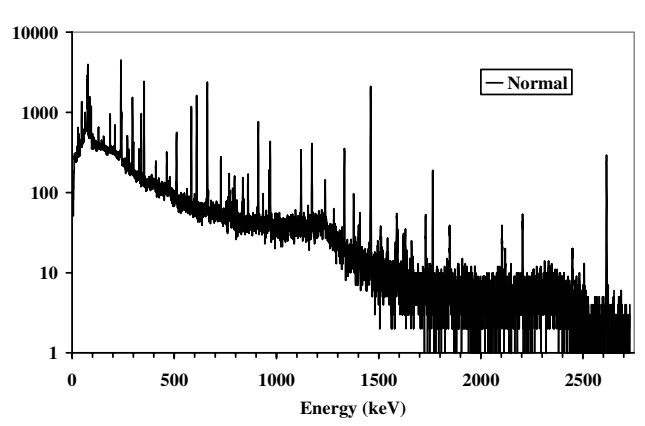

(a)

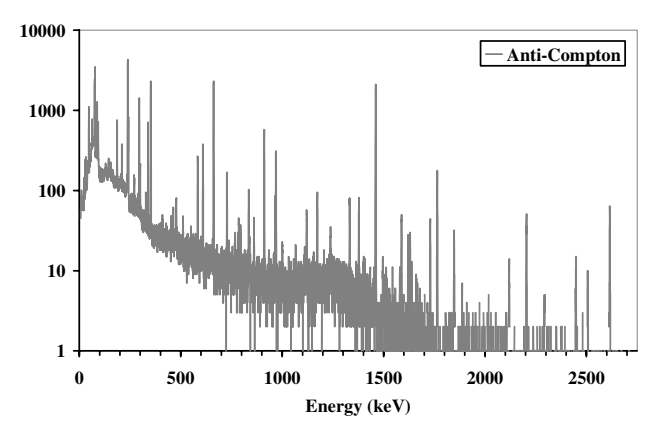

(b)

Figure 4. Spectra obtained for the measurement of particles of fluvial water (ashes) during $80000 \mathrm{~s}$.

Thanks to the anti-Compton system we are able to:

- determine more precisely activities of ${ }^{7} \mathrm{Be}( \pm 26 \% \rightarrow \pm 16 \%)$ and ${ }^{54} \mathrm{Mn}( \pm 11 \% \rightarrow \pm 9 \%)$, and to a smaller extent ${ }^{210} \mathrm{~Pb},{ }^{234} \mathrm{Th}$ and ${ }^{137} \mathrm{Cs}$,

- decrease the detection limits for ${ }^{241} \mathrm{Am},{ }^{57} \mathrm{Co}$ and ${ }^{58} \mathrm{Co}$ in similar proportions to the case of mussel sample,

- determine significant values instead of detection limits for ${ }^{65} \mathrm{Zn}(\leq 1.64 \mathrm{~Bq} / \mathrm{kg} \rightarrow 0.49 \pm$ $0.26 \mathrm{~Bq} / \mathrm{kg}$ ).

As already shown in the previous paragraph using the Compton-suppressed spectrum to analyze ${ }^{60} \mathrm{Co}$ leads to an underestimation of its activity: $1.8 \pm 0.2 \mathrm{~Bq} / \mathrm{kg}$ instead of $8.2 \pm 0.4 \mathrm{~Bq} / \mathrm{kg}$ because of the very significant count losses of this multi-gamma emitter.

\subsection{Aerosols filters with ${ }^{7} \mathrm{Be}$}

An aerosol filter has been measured during 320000 s. Spectra on Fig 5a have two components: the large photopeak of the ${ }^{7} \mathrm{Be}\left(8000 \mu \mathrm{Bq} / \mathrm{m}^{3}\right)$ and its Compton continuum which dominates at energies lower than $500 \mathrm{keV}$. The second component is the cosmic background which can be very well observed between 500 and $2700 \mathrm{keV}$ in the absence of any rays.

${ }^{7} \mathrm{Be}$ Compton continuum is largely reduced $(\mathrm{SF}=6.3)$ and the annihilation line is reduced in a spectacular way by a factor 37 on the peak areas. This reduction is indicative of the anti-cosmic aspect of the anti-Compton device. As shown on Fig 5b the reduction of the cosmic-ray induced background let appear the ${ }^{137} \mathrm{Cs}$ peak at $661.7 \mathrm{keV}$ : we can thus determine a significant value for this radionuclide.

Concerning $\beta^{+}$emitter ${ }^{22} \mathrm{Na}$ because of the detection of both $511 \mathrm{keV}$ gamma in coincidence in $\mathrm{NaI}$ and GeHP detectors, the analysis of the Compton-suppressed spectrum would lead to a large

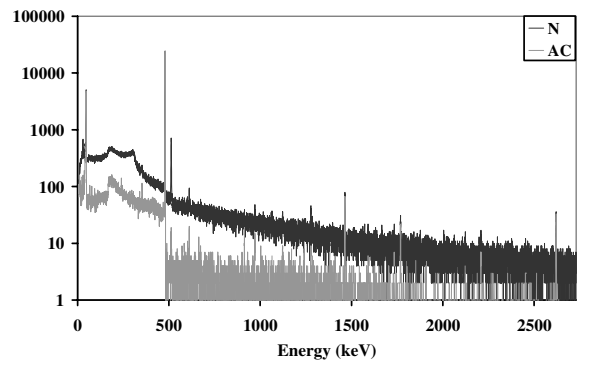

(a)

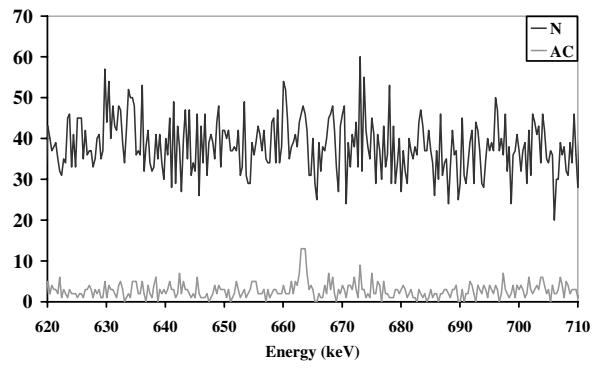

(b)

Figure 5. Spectra obtained for the measurement of an aerosol filter during $320000 \mathrm{~s}$. 
underestimation of the activity of this radionuclide: $0.11 \pm 0.06 \mu \mathrm{Bq} / \mathrm{m}^{3}$ instead of $0.83 \pm$ $0.16 \mu \mathrm{Bq} / \mathrm{m}^{3}$.

With the anti-Compton system we can:

- determine more precisely activities of ${ }^{40} \mathrm{~K}( \pm 55 \% \rightarrow \pm 51 \%)$

- decrease the detection limits for ${ }^{241} \mathrm{Am},{ }^{57} \mathrm{Co}$ and ${ }^{58} \mathrm{Co}$ (divided by 3.5 )

- obtain significant value instead of detection limit for ${ }^{137} \mathrm{Cs} \quad\left(\leq 0.34 \mu \mathrm{Bq} / \mathrm{m}^{3} \rightarrow 0.158 \pm\right.$ $\left.0.053 \mu \mathrm{Bq} / \mathrm{m}^{3}\right)$.

\section{CONCLUSIONS}

The use of Compton suppression is a competitive tool in trace measurements of some radionuclides in environmental samples where natural radioactivity and/or presence of artificial radionuclide with high activity give rise to an important Compton continuum.

We have shown on different kinds of environmental samples that : depending on the sample and the radionuclide, the uncertainties on low activity values can be improved, the detection limits can be significantly reduced (up to a factor 3.5 for ${ }^{58} \mathrm{Co}$ ) and thus significant values can be determined instead of detection limits (e.g. for ${ }^{137} \mathrm{Cs}$ or ${ }^{65} \mathrm{Zn}$ ).

However this tool has to be used with care depending on the studied radionuclides, as shown in the case of ${ }^{60} \mathrm{Co}$ and ${ }^{22} \mathrm{Na}$.

\section{References}

[1] Landsberger S. and Pashev S., J. Radioanal. Nucl. Chem. 202 (1996) 201-224.

[2] “Compton Suppression ... Made Easy", Canberra Application Note, AN-D-8901.

[3] Report CIRP-CTHIR-GTN5 n 41 (1990).

[4] Heusser G., Nucl. Instr. and Meth. B 58 (1991) 79. 